\title{
Endoscopic Closure of a Postoperative Duodenal Fistula
}

\author{
Tammy L. Kindel, MD, PhD, Caitlin A. MacGregor, BA, Rajesh N. Keswani, MD, \\ David M. Mahvi, MD \\ Department of Surgery (Drs. Kindel, MacGregor, Mahvi) and Department of Medicine (Dr. Keswani), Northwestern \\ University Feinberg School of Medicine, Chicago, IL, USA.
}

\begin{abstract}
Introduction: Bouveret syndrome is a rare presentation of gallstone ileus characterized by passage of a gallstone through a cholecystoenteric fistula and impaction of the gallstone within the duodenum, causing proximal small bowel obstruction. Most patients require surgery with duodenotomy and stone extraction. Unfortunately, duodenal surgery has a high risk for surgical complications, including suture-line leaks and fistula formation. Fistulas that fail to close with conservative management, and those complicated by electrolyte and fluid disturbances, require surgical correction. Recently, overthe-scope clip devices have been used successfully for enteric fistula closure.
\end{abstract}

Case Description: We present the case of a 33-year-old man with a postsurgical duodenal leak after duodenorrhaphy as surgical treatment for Bouveret syndrome. Two weeks after surgery, the patient underwent successful over-the-scope clip closure of a high-output duodenal fistula, requiring no subsequent endoscopic or operative fistula management.

Discussion: Over-the-scope clips appear promising for successful closure of postsurgical duodenal fistulas that persist despite conservative management and optimized nutritional status.

Key Words: Duodenal fistula, Over-the-scope clip, OTSC, Bouveret syndrome, Fistula closure.

Citation Kindel TL, MacGregor CA, Keswani RN, Mahvi DM. Endoscopic closure of a postoperative duodenal fistula. CRSLS e2014.00368. DOI: 10.4293/CRSLS.2014.00368.

Copyright (C) 2014 SLS This is an open-access article distributed under the terms of the Creative Commons Attribution-Noncommercial-ShareAlike 3.0 Unported license, which permits unrestricted noncommercial use, distribution, and reproduction in any medium, provided the original author and source are credited.

Address correspondence to: Tammy L. Kindel, MD, PhD, University of Nebraska Medical Center, Division of General Surgery, 983280 Nebraska Medical Center, Omaha, NE 68198-3280, USA. Telephone: 402-559-8990, E-mail: tammy_venema@yahoo.com

\section{INTRODUCTION}

Patients undergoing duodenal surgery are unfortunately at high risk for enteric leakage because of the constant exposure of a suture repair to intraluminal digestive enzymes. Adequate closed-suction drainage of the periduodenal area placed at the time of surgery can convert an abdominal catastrophe and sepsis into a controlled duodenal fistula. The likelihood of spontaneous duodenal fistula closure with aggressive sepsis control and total parenteral nutrition (TPN) varies widely in the literature, from $28 \%$ to $92 \% .^{1-4}$ Even in the setting of spontaneous closure, conservative management can require months of TPN, which carries the associated risks of parenteral nutrition and an indwelling central line, in addition to the significant electrolyte disturbances with high-output fistulas. Furthermore, persistent duodenal fistulas require re- exploration for definitive management, frequently using a defunctionalized Roux-en-Y duodenojejunostomy. 5 There are reports of endoscopic management of postsurgical duodenal fistulas, including stenting, chemical injection, and clip closure. ${ }^{6-9}$ We report here the use of an overthe-scope clip (OTSC) for closure of a high-output duodenal fistula in a patient who had undergone duodenorrhaphy as surgical treatment for Bouveret syndrome.

\section{CASE DESCRIPTION}

A 33-year-old, morbidly obese man, with a medical and surgical history of biliary colic, gastroesophageal reflux disease, and an appendectomy, presented to an outside hospital with symptoms of bilious emesis and postprandial epigastric pain for the past 6 days. He had no other associated symptoms, with normal vital signs and benign 
results on abdominal examination. Laboratory values were within normal limits except for slightly elevated alkaline phosphatase (106 U/L). Radiographic imaging in the emergency department included contrast-enhanced computed tomography of the abdomen and pelvis, which revealed air in the biliary tree and a proximal small bowel obstruction with a transition point at the third portion of the duodenum due to a $4.8-\mathrm{cm}$ intraluminal gallstone consistent with Bouveret syndrome (Figure 1).

After hospital admission and nasogastric decompression, the decision was made to take the patient to the operating room the following day for open abdominal exploration and gallstone removal. At the time of exploration, the gallstone was confirmed to be within the second portion of the duodenum. A 3-cm longitudinal duodenotomy was performed. The stone was fractured with forceps and fully removed. The duodenotomy was closed longitudinally in 1 layer and covered with omentum and fibrin glue. One Jackson-Pratt (JP) drain was placed for closed-suction drainage in the event of staple-line dehiscence, and the nasogastric tube was left in place for antegrade drainage. The patient was initiated on TPN on postoperative day (POD) 1. Unfortunately, on POD 5, bilious JP drain output

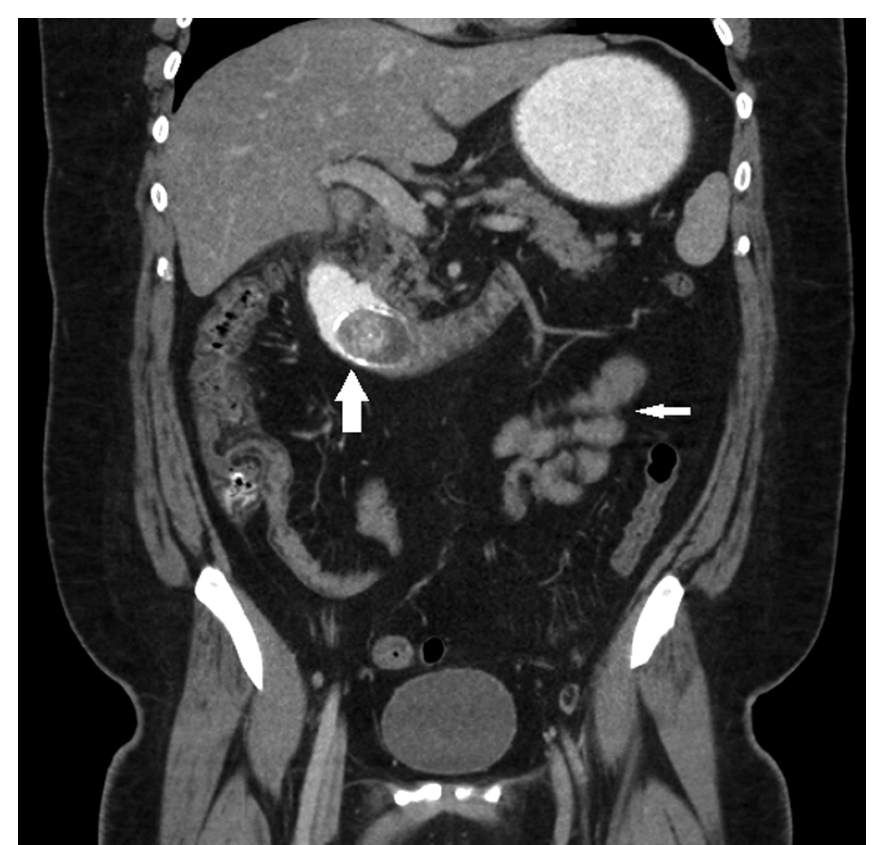

Figure 1. Computed tomographic coronal section showing a contrast-distended stomach and proximal duodenum. An intraluminal gallstone was found within the second portion of the duodenum (large white arrow), causing a proximal small bowel obstruction with decompressed distal small bowel loops (small white arrow).

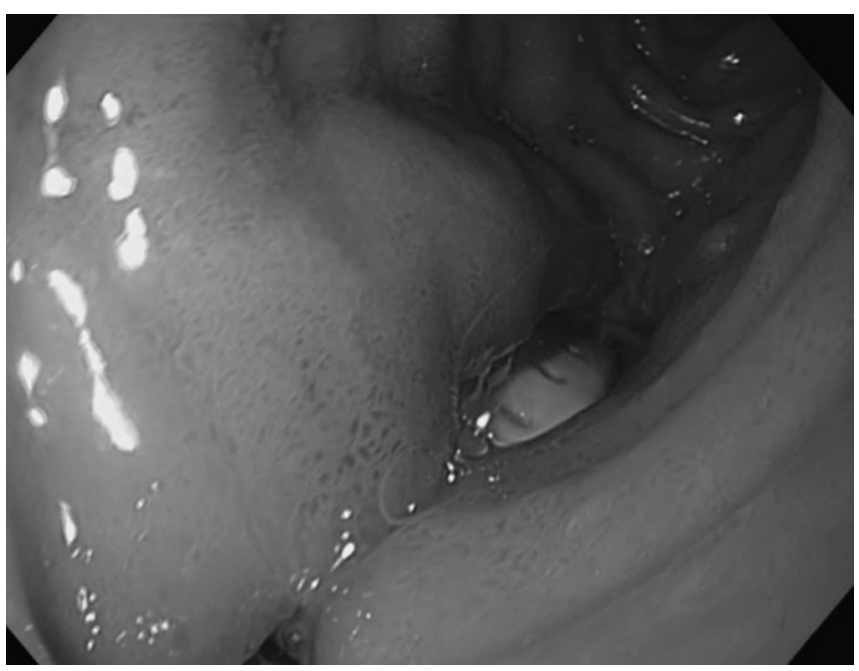

Figure 2. Endoscopic view of a suture-line dehiscence in the second portion of the duodenum, with the Jackson-Pratt drain visible within the base.

was noted. The patient developed leukocytosis to $20,500 / \mu \mathrm{L}$ with mild abdominal pain over the next 48 hours. The patient was started on piperacillin/tazobactam empirically, and computed tomography was performed, showing drainage of contrast into the JP drain with no passage of contrast past the duodenorrhaphy. There were a few scattered nonenhancing fluid collections but no free extravasation of contrast. The patient was then transferred on POD 7 to our tertiary care center for further management of his duodenal leak.

On transfer, the patient was hemodynamically stable, without peritoneal signs. His white blood cell count normalized over 3 days on antibiotic therapy. His JP drain output was consistent with a controlled duodenal leak, with output of approximately $1,500 \mathrm{~cm}^{3} /$ day. After consultation with our interventional gastroenterology service, the patient was taken to the endoscopy suite on POD 15 for evaluation of the suture line to rule out a severe or complete duodenal obstruction as the cause of the duodenal fistula. Furthermore, if the distal duodenal lumen was patent, attempts would be made during OTSC placement to close this high-output fistula. Endoscopy was performed with carbon dioxide insufflation. A mediumsized cholecystoduodenal fistula was observed at the duodenal bulb. A large suture line dehiscence ( 20 and $5 \mathrm{~mm}$ ) was found in the second portion of the duodenum, with a patent distal lumen (Figure 2). The JP drain was visualized within the base of the suture line dehiscence and repositioned under direct endoscopic visualization. As shown in Figure 3, an Ovesco (Los Gatos, CA) OTSC 


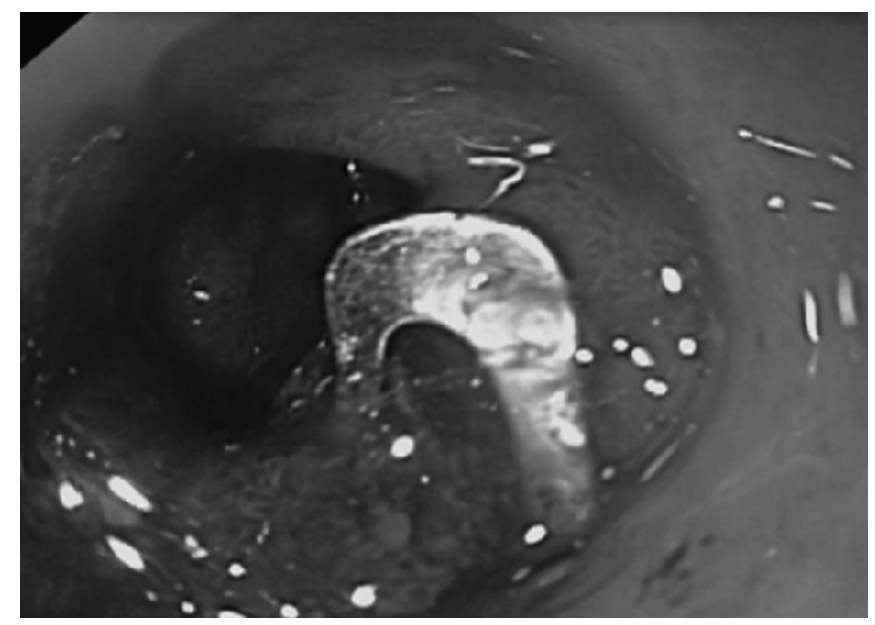

Figure 3. Endoscopic successful deployment of an Ovesco overthe-scope clip.

(12-mm cap length, 6-mm cap depth, type t) was successfully deployed, with full closure of the suture line fistula. The nasogastric tube was removed at the time of the procedure and not replaced.

After OTSC placement, the patient was kept nil per os and on TPN while successful fistula closure was determined. The JP drain output decreased over the following week to $15 \mathrm{~cm}^{3} /$ day at the time of hospital discharge on POD 23. One week later, the patient was seen in the clinic, with minimal drain output. An upper gastrointestinal series confirmed closure of the duodenal leak, with no extravasation of barium contrast. The patient was started on a liquid diet, which was advanced over the next week to a general diet, with no change in JP drain output, at which point the drain was removed uneventfully.

\section{DISCUSSION}

Bouveret syndrome presents as a gastric outlet obstruction due to impaction of a gallstone at the distal stomach or duodenum after the development of a cholecystoenteric fistula. This case involved an atypical patient for Bouveret syndrome, being both male and young. Bouveret syndrome is exceptionally rare, representing $<10 \%$ of enteric gallstone obstructions, and generally occurs in elderly women. ${ }^{10,11}$ Similar to what we experienced in our patient, the obstructing gallstone in Bouveret syndrome is on average $4.6 \mathrm{~cm}$, and patients commonly have histories of chronic cholecystitis. ${ }^{12,13}$

Surgical treatment is required for $91 \%$ of patients with Bouveret syndrome. ${ }^{13}$ This can be done as a 1-stage repair with enterotomy, cholecystectomy, and cholecystoduode- nal fistula repair. More commonly, enterotomy with stone extraction only is performed at the time of the initial surgery. Although the necessity has been debated in the literature, a second surgery for cholecystoduodenal fistula repair can be done electively if the patient is symptomatic, young, and/or of acceptable surgical risk. ${ }^{13}$

Endoscopy as primary treatment is successful in only $9 \%$ to $18 \%$ of patients with minimal morbidity and mortality. ${ }^{13,14}$ However, unless a patient exhibits signs or symptoms mandating immediate surgical exploration, endoscopy should be attempted by a skilled endoscopist. When successful, endoscopic extraction affords the patient a significantly reduced complication profile compared with urgent or emergent surgical exploration. The surgical mortality rate for treatment of Bouveret syndrome varies from $5 \%$ to $24 \%$, although higher mortality rates likely reflect the older age and multiple comorbidities within this patient population. ${ }^{13,14}$ Endoscopic attempts at removal include endoscopic extraction or mechanical lithotripsy, with awareness that stone fragments may progress further downstream, presenting as classic gallstone ileus. ${ }^{15,16}$ However, a jejunal or ileal enterolithotomy is more desirable than a duodenotomy and stone extraction.

Traditionally, surgery has been the only definitive option if a duodenal fistula fails to close spontaneously with conservative management. The OTSC is made of an elastic, shape-memory nitinol alloy, making this a highly attractive device for closure of fibrotic and chronically inflamed tissue, often encountered in enteric fistulas. ${ }^{17}$ An OTSC, compared with a conventional endoscopic clip, allows the closure of larger defects because of its larger jaw size. There are multiple reports of the use of OTSC devices for gastrointestinal bleeding, enteric fistulas, and gastrointestinal perforations. ${ }^{8,18-20}$ However, specific application for duodenal fistulas is limited in the literature. Bini et al ${ }^{9}$ described the successful closure of a highoutput postsurgical duodenal fistula in a 78-year-old patient who had undergone emergent gastric resection for bleeding peptic ulcer disease. Manta et $\mathrm{al}^{8}$ described a series of 12 patients undergoing OTSC placement for a variety of reasons, 3 of whom had duodenal fistulas. Two fistulas were at a duodenojejunal anastomosis, and the third was a suture line leak after attempted surgical repair of an endoscopic perforation. One of the 3 patients failed OTSC fistula closure and required subsequent endoluminal stent placement.

This report describes additional experience with the successful use of an OTSC for closure of a postsurgical duodenal fistula. Although still a new and emerging technol- 
ogy, OTSCs appear promising for successful closure of duodenal fistulas that persist despite conservative management and optimized nutritional status. Further investigation of this device should include analysis of patients who are good candidates for OTSC management, which may be based on fistula size, duration, etiology, and anatomic location.

\section{References:}

1. Malangoni MA, Madura JA, Jesseph JE. Management of lateral duodenal fistulas: a study of fourteen cases. Surgery. 1981; 90:645-651.

2. Williams NM, Scott NA, Irving MH. Successful management of external duodenal fistula in a specialized unit. Am J Surg. 1997;173:240-241.

3. Garden OJ, Dykes EH, Carter DC. Surgical and nutritional management of postoperative duodenal fistulas. Dig Dis Sci. 1988;33:30-35.

4. De Backer A, Fetelian D, Carpentier Y, Willems G. Postoperative duodenal fistulas. A report of 23 cases with emphasis on the surgical treatment by the Roux-en-Y operation. Acta Chir Belg. 1985;85:185-191.

5. Mulholland M. Greenfield's Surgery: Scientific Principles and Practice. 5th ed. Philadelphia, PA: Lippincott Williams \& Wilkins; 2011.

6. Vezakis A, Fragulidis G, Nastos C, Yiallourou A, Polydorou A, Voros D. Closure of a persistent sphincterotomy-related duodenal perforation by placement of a covered self-expandable metallic biliary stent. World J Gastroenterol. 2011;17:4539-4541.

7. Ozdil B, Yamak YZ, Kece C, Cebi K. Successful endoscopic therapy of postoperative duodenal fistula by lipiodol injection: a new therapeutic approach. Minim Invasive Ther Allied Technol. 2011;20:193-196.

8. Manta R, Manno M, Bertani H, Barbera C, et al. Endoscopic treatment of gastrointestinal fistulas using an over-the-scope clip (OTSC) device: case series from a tertiary referral center. Endoscopy. 2011;43:545-548.

9. Bini R, Coppola F, Recchia S, Fusca M, Gaia S, Leli R. Endoscopic treatment of postgastrectomy duodenal fistula with an over-the-scope clip. Surg Innov. 2011;18:102-104.
10. Mavroeidis VK, Matthioudakis DI, Economou NK, Karanikas ID. Bouveret syndrome-the rarest variant of gallstone ileus: a case report and literature review. Case Rep Surg. 2013;2013: 839370 .

11. Nickel F, Muller-Eschner MM, Chu J, von Tengg-Kobligk H, Muller-Stich BP. Bouveret's syndrome: presentation of two cases with review of the literature and development of a surgical treatment strategy. BMC Surg. 2013;13:33.

12. Reinhardt SW, Jin LW, Pitt SC, Earl TM, Chapman WC, Doyle MB. Bouveret's syndrome complicated by classic gallstone ileus: progression of disease or iatrogenic? J Gastrointest Surg. 2013; $17: 2020-2024$.

13. Lowe AS, Stephenson S, Kay CL, May J. Duodenal obstruction by gallstones (Bouveret's syndrome): a review of the literature. Endoscopy. 2005;37:82-87.

14. Cappell MS, Davis M. Characterization of Bouveret's syndrome: a comprehensive review of 128 cases. Am J Gastroenterol. 2006;101:2139-2146.

15. Katsinelos P, Kimiropoulos S, Tsolkas P, et al. Successful treatment of duodenal bulb obstruction caused by a gallstone (Bouveret's syndrome) after endoscopic mechanical lithotripsy. Surg Endosc. 2002;16:1363.

16. Alsolaiman MM, Reitz C, Nawras AT, Rodgers JB, Maliakkal BJ. Bouveret's syndrome complicated by distal gallstone ileus after laser lithotripsy using holmium:YAG laser. BMC Gastroenterol. 2002;18:15.

17. Jayaraman V, Hammerle C, Lo SK, Jamil L, Gupta K. Clinical application and outcomes of over the scope clip device: initial US experience in humans. Diagn Ther Endosc. 2013;2013:381873.

18. von Renteln D, Denzer UW, Schachschal G, Anders M, Groth S, Rosch T. Endoscopic closure of GI fistulae by using an over-thescope clip (with videos). Gastrointest Endosc. 2010;72:1289-1296.

19. Nishiyama N, Mori H, Kobara H, et al. Efficacy and safety of over-the-scope clip: including complications after endoscopic submucosal dissection. World J Gastroenterol. 2013;19:2752-2760.

20. Disibeyaz S, Koksal AS, Parlak E, Torun S, Sasmaz N. Endoscopic closure of gastrointestinal defects with an over-the-scope clip device. A case series and review of the literature Clin Res Hepatol Gastroenterol. 2012;36:614-621. 